\title{
Recovery and maintenance of NESTIN expression in umbilical cord-MSC using a novel culture medium
}

\author{
Yuncheng Liu ${ }^{1 \dagger}$, Feidi Xiao ${ }^{2 \dagger}$, Xiang Hu², Zan Tang², Zeqin Fu², Xiao Liang², Guifang Zeng², Weijie Zeng', \\ Yan Liao' ${ }^{2}$ Yuan Ren' ${ }^{1}$ Zhiyu Liu', Hao Peng ${ }^{1}$, Qiuhong Mei $^{3}$ and Muyun Liu ${ }^{1 *}$
}

\begin{abstract}
Mesenchymal stem cells (MSC) are a popular candidate in cellular therapy for many diseases. MSCs are well known by their feature of self-renewal and their differentiation potential. NESTIN is a cytoskeletal protein expressed in MSC that functions directly in cell proliferation and differentiation. Here, we demonstrated that adding UltraGRO, a medium supplement, could maintain and partially recover the expression of NESTIN in human umbilical cord derived MSCs (UC-MSCs). Furthermore, the UC-MSCs cultured with UltraGRO showed a better immunomodulation ability in a colitis mouse model compared with those cultured in other types of media. This indicates that the use of novel culture medium benefits the maintenance of NESTIN expression and NESTIN may be one of the vital factors that regulates the performance of MSCs.
\end{abstract}

Keywords: NESTIN, UC-MSC, Culture medium, UltraGRO

\section{Key points}

1. NESTIN affects the proliferation and differentiation of mesenchymal stem cells, however, its expression decreases over time with frequent, iterated passaging.

2. Using a novel culture medium by adding UltraGRO can sufficiently maintain and recover the expression of NESTIN in umbilical cord MSCs for up to 10 passages.

3. The UC-MSCs cultured using this novel medium have better immunomodulatory ability in colitis mice models.

\footnotetext{
*Correspondence: liumuyun@nlelpct.com

†Yuncheng Liu and Feidi Xiao contributed equally to this work

${ }^{1}$ National-Local Associated Engineering Laboratory for Personalized Cell

Therapy, Nanshan, Shenzhen, Guangdong, People's Republic of China

Full list of author information is available at the end of the article
}

\section{Introduction}

Since Friedenstein and co-workers first identified the differentiation potential of bone marrow cells in 1987, numerous studies on stem cells have been conducted in various fields. These studies have demonstrated that "bone marrow fibroblasts" and their descendants originated from non-hematopoietic stem cells and could differentiate into mesenchymal cells of other tissues (Friedenstein et al. 1987). So-called "mesenchymal stem cells" (MSCs) are widely studied mainly due to their feature of self-renewal and their differentiation potential (Uder et al. 2018). The International Society of Cell Therapy (ISCT) specifically defines human MSC by three criteria. The first is that MSCs can adhere to plastic surfaces, which is utilized to easily isolate cells from tissues under standardized culture conditions. Second, MSCs from different sources must have a similar combination of surface markers, that is, up to $95 \%$ of the MSC population expresses CD105, CD73, and CD90. However, less than 2\% of MSC may express CD45, CD34, CD14 or CD11b, CD79 or CD19 and HLA-DR (Dominici et al. 2006). 
Lastly, MSCs must feature a robust differentiation potential, and must be able to differentiate into at least three different lineages in vitro, such as osteoblasts, adipocytes, and chondrocytes (Uder et al. 2018). These criteria have become a gold standard in the stem cell therapy industry, and are used to confirm MSC during all manufacturing processes, from cell isolation to stimulated culture and finally to large-scale expansion.

Despite MSCs first being identified from bone marrow, they have also been isolated from many other tissues and organs, such as muscle, thymus, pancreas, adipose, dental pulp, umbilical cords, and placenta (Ferrari et al. 1998; Seo et al. 2004; Zuk et al. 2001). MSCs are distributed in many different organs likely because MSCs are generated in and can migrate via large and small blood vessels. This may also be relevant to their residential preference in perivascular niches throughout post-natal organisms (da Silva Meirelles et al. 2006). Accordingly, MSCs are manufactured by adipose tissue, umbilical cords, and placenta, which are commonly treated as clinical wastes, under current Good Manufacturing Practice (cGMP) in the stem cell therapy industry. However, flow cytometry analyses revealed that MSCs between different sources and species have similar but not identical surface markers and functions (Donnenberg and Ulrich 2013). Thus, investigations of the expression of surface markers and morphology were performed to monitor the association of effects on variability in terms of isolation yield, proliferation rates, and expansion ability (Kern et al. 2006; Oedayrajsingh-Varma et al. 2006; Seo et al. 2005).

NESTIN, a neuroepithelial stem cell protein, is also a cytoskeletal protein expressed in stem cells (Lendahl et al. 1990; Mignone et al. 2004). It is believed that NESTIN functions in the stem cell processes of self-renewal, proliferation, differentiation, and migration (Bernal and Arranz 2018; Kulkarni et al. 2017; Mignone et al. 2004). According to the gene sequence and protein structure homology, NESTIN is classified as a type VI intermediate filament that contributes to cytoskeleton constitution (Bernal and Arranz 2018). The constitutive expression of NESTIN by undifferentiated MSC is regarded as a marker of the "multi-differentiated" state in which cells can retain their neuronal differentiation property (Tondreau et al. 2004). Moreover, NESTIN expression is developmentally regulated because it is inversely correlated with cell differentiation (Wiese et al. 2004). NESTIN is downregulated by the transition from proliferating neural stem cells to post-mitotic neurons with specific stimulus (Wiese et al. 2004; Zimmerman et al. 1994). Many recent studies have indicated that NESTIN may be an important reporter of cell state related to proliferation and differentiation potential of MSCs (Bernal and Arranz 2018; Lu et al. 2019; Wong et al. 2014).
However, NESTIN is not currently a common selective marker for MSCs in either ISCT criteria or the stem cell therapy industry. MSCs are prominent due to their self-renewal potential, which allows the propagation of MSCs in vitro under specific isolation and cultivation procedures (Uder et al. 2018). Since MSC application for clinical use has strict requirements for sufficient cell number and consistent cell quality along the whole cultivation process, the in vitro expansion of MSCs has been extensively investigated. These studies revealed that the isolation, culture, expansion procedures and cell differentiation during the manufacturing process are possible factors resulting in the inconsistency (Lodie et al. 2002; Uder et al. 2018). The conventional MSC culture protocol as described in numerous studies uses Dulbecco's modified Eagle's medium (DMEM) with 10\% fetal bovine serum (FBS). Since frequent iterated passaging for MSC would lead to cell aging and loss of stemness, human MSC culture is confined prior to the 6th passage in order to maintain the unrestricted differentiation capacities (Bonab et al. 2006; Halfon et al. 2011).

The ex vivo expanded MSCs have a potent immunomodulatory function in the treatment of immune disorders. Many inflammation-related cytokines, such as interleukin-6 (IL-6), interleukin-1 $\beta$ (IL-1 $\beta$ ), indoleamine 2,3-dioxygenase (IDO) and prostaglandin E2 (PEG2), can be modulated in the presence of MSCs (Chinnadurai et al. 2014; Lara et al. 2017; Wang et al. 2017). The clinical potential of MSC transfusion has been explored in several animal models of immune disorders including autoimmune encephalitis, rheumatoid arthritis, transplant rejection and Crohn's disease (Ren et al. 2012; Uccelli et al. 2008). In this study, we monitored the NESTIN expression in umbilical cord MSCs (UC-MSCs) in vitro for up to ten passages using three different types of culture media. Moreover, we verified the immunomodulatory ability of UC-MSCs in colitis mice models.

\section{Materials and methods Isolation and culture of human UC-MSCs}

Institutional review board approval from the Shenzhen Integrated Cell Bank was obtained for all procedures. Fresh umbilical cords (UC) were collected for scientific study from three healthy donors who were informed and consented. Mesenchymal tissue was scraped from Wharton's jelly after blood vessels were removed (Devito et al. 2019). After cutting it into pieces, the tissue was centrifuged at $600 \mathrm{~g}$ for $10 \mathrm{~min}$ at room temperature. Tissue was then washed with $0.9 \%$ saline and cultured at $37{ }^{\circ} \mathrm{C}$ with $5 \% \mathrm{CO}_{2}$ in serum-free Dulbecco's modified Eagle's medium (DMEM). The primary UC-MSCs (P0) were obtained after 10 days of culture. A sufficient number of MSCs from the same source was equally divided into 
three groups in order to ensure the same starting conditions. During the 10-passage culture, UC-MSCs were plated at the density of $5 \times 10^{3} \mathrm{cells} / \mathrm{cm}^{2}$ into flasks for each group and cultured at $37{ }^{\circ} \mathrm{C}$ and $5 \% \mathrm{CO}_{2}$. Three culture medium supplements were applied for comparison including fetal bovine serum (FBS), fetal bovine serum substitute (FS) (TBD, China), and UltraGRO-Advanced (Helios Bioscience, UK); the base medium for all cultures was DMEM/F-12 (Gibco, US). Base culture ingredients were DMEM and $10 \%$ FBS, which is the conventional culture medium. The second type of media was DMEM with $2 \%$ TBD FS and 1\% GlutaMax, and the third was DMEM with 5\% UltraGRO-advanced and 1\% GlutaMax.

\section{Induction assay for UC-MSC differentiation}

Based on a published procedure, the UC-MSCs were induced to differentiate into osteogenic, adipogenic and chondrogenic stem cells in vitro (Lei et al. 2013).

\section{UC-MSC assay proliferation}

To investigate the effects of different culture systems, 10th passage UC-MSCs were used to perform the following experiments. The 10th passage UC-MSCs of the three groups were passaged and the cell counts were recorded at the 6th, 12th, 24th, and 48th hours of culture. The expansion ratio was calculated by dividing the total cell count by the initial plated cell number. Moreover, 10th passage UC-MSCs of the three groups were also treated with $200 \mathrm{ng} / \mathrm{ml} \mathrm{IFN- \gamma}$ for $4 \mathrm{~h}$. IDO and PGE2 were detected in the culture supernatant using an ELISA from BioLegend (San Diego, CA).

\section{Flow cytometry analyses}

Flow cytometry analyses were performed using a FACSaria ${ }^{\mathrm{TM}}$ III cytometer (BD Bioscience, San Jose, CA) and the data were analyzed with the FlowJo7.5 (Treestar, Ashland, OR) software packages. In order to identify UC-MSCs, Antihuman CD29-PE (MAR4), CD73-PECyTM7 (AD2), CD90-FITC (5E10), CD105-APC (266), CD34-PE (563), CD45-FITC (HI30), CD14-FITC (M5E2), CD79 $\alpha$-APC (HM47) and HLA-DR-PreCP (G46-6) antibodies, along with the corresponding isotype control antibodies were purchased from BD Pharmingen. To monitor the NESTIN expression, antihuman NESTINAPC (IC1259A) antibody along with the corresponding isotype control antibody was also purchased from R\&D Pharmingen. As NESTIN is a protein of the cytoskeleton, cell fixation and permeabilization were performed before cell staining for flow cytometry. Sufficient MSCs from each group at each passage were harvested for flow cytometry analyses.

\section{Quantitative PCR assay}

UC-MSC samples were collected at each time point and stored at $-80{ }^{\circ} \mathrm{C}$. RNA extraction was then performed using the RNeasy Mini Kit from QIAGEN. The forward and reverse primers for qPCR were designed for NESTIN detection (F: CTCCAAGAATGGAGGCTG TAGGAA, R: CCTATGAGATGGAGCAGGCAAGA). QuantiFast ${ }^{\circledR}$ SYBR ${ }^{\circledR}$ Green PCR Kit from QIAGEN was used to perform qPCR.

\section{Experimental colitis induced by TNBS}

All of the animal procedures were reviewed and approved by the Beike Animal Care and Use Committee. For inducing colitis in 9-week-old male BALB/c mice, we referred to a previously published protocol (Wirtz et al. 2007). On day 1 , mice were smeared with $200 \mu \mathrm{l}$ of a pre-sensitization solution of trinitrobenzene sulfonic acid (TNBS; Sigma) on their backs. On day 8 , mice were divided into ten groups ( 8 mice/group) and fasted (but allowed to drink ad libitum) for $24 \mathrm{~h}$. On day 9 , mice were weighed and treated intrarectally with $150 \mu \mathrm{l} 3 \%$ TNBS in saline (eight groups) and no solution (blank control, two groups). At $10 \mathrm{~h}$ post TNBS injection, animals were transplanted i.p. with $500 \mu \mathrm{l}$ saline, or $4 \times 10^{6} \mathrm{MSCs}$ from the three groups that were suspended in $500 \mu \mathrm{l}$ saline. Colons were collected from the caecum to the anus on day- 1 , day 0 , day 2 , day 4 , day 6 and day 10 after TNBS injection.

\section{Cytokine assays}

The culture supernatant of 10th passage UC-MSCs was used to detect the cytokine production of IDO (DY6030-05) and PGE2 (KGE004B) with an ELISA from R\&D system Inc. Mouse serum was used to detect the cytokines. IL-6 (431304) and IL-1 $\beta$ (437003) were analyzed using an ELISA from BioLegend (San Diego, CA) and SOD (MAB3419-SP) using an ELISA from R\&D system Inc.

\section{Statistical analysis}

Statistical comparisons were performed using the two-tailed Student's $t$-test (between two groups) or a one-way analysis of variance (ANOVA). $\mathrm{P}<0.05$ was considered to represent a significant difference. Analysis and graphing were performed using WPS office software.

\section{Results}

The three different culture conditions used to obtain mesenchymal stem cells (MSCs) for the comparisons were: DMEM-F12 with $10 \%$ fetal bovine serum (FBS), DMEM-F12 with $10 \%$ fetal bovine serum substitute 
(FS), and DMEM-F12 with 5\%UltraGRO-Advanced. UC-MSCs were harvested at various passages throughout the culture period, and the immunophenotype of the MSCs was investigated via quantitative flow cytometry (Uder et al. 2018). Flow cytometric analyses revealed that the UC-MSCs at the 10th passage in all three culture conditions expressed representative surface markers, including CD29, CD73, CD90, and CD105, but not CD34, CD45, CD14, CD79, or HLADR (Fig. 1a). Cells from the 2nd to 9th passage also had the same surface marker repertoire (data not shown).To compare the differentiation potentials, the UC-MSCs in different culture conditions were induced to differentiate into adipogenic, osteogenic, or chondrogenic lineages. UC-MSCs in all three culture conditions at the 10th passage were successfully induced to differentiate into adipogenic, chondrogenic, and osteogenic cells (Fig. 1b), and the UC-MSCs from the 1st to 9th passage had the same differentiation ability. The results suggest that all three culture conditions could sustain the surface marker repertoire and differentiation potential of the UC-MSCs from the 1st to 10th passage.

We next examined the percentage of $\mathrm{NESTIN}^{+} \mathrm{UC}$ MSCs by flow cytometry analysis (Fig. 2a, b) and the NESTIN expression level in UC-MSCs by quantitative reverse transcription PCR (RT-qPCR) in three different culture conditions in each passage. The ratio of NESTIN $^{+}$UC-MSCs could be stably maintained before the 6th passage in all three culture conditions. However, the percentage of NESTIN ${ }^{+}$UC-MSCs significantly decreased starting at the 7 th passage in the other two culture conditions compared with UltraGRO-medium (Fig. 2c). Consistently, NESTIN in UCMSCs in three different culture conditions had a similar trend with the exception of expression level (Fig. 2d). NESTIN in UltraGRO-medium was expressed at a relatively higher level than the others. Despite the fact that UltraGRO-medium could sustain the percentage of $\mathrm{NESTIN}^{+}$UC-MSCs, we further investigated whether the shift of UltraGRO-medium could recover the expression of NESTIN in UC-MSCs in the other two culture conditions. The UC-MSCs were initially cultured in FBS-medium or TBD-FS-medium, and then changed to UltraGRO-medium at the 4th passage. We found that the percentage of NESTIN ${ }^{+}$UC-MSCs increased after changing medium compared with the UC-MSCs cultured using consistent culture conditions. If we changed the culture medium back to FBS-medium or TBD-FS-medium at the 6th passage, the percentage of NESTIN ${ }^{+}$UC-MSCs declined significantly and increased significantly when the medium was changed back to UltraGRO-medium at the 8th passage (Fig. 2e, g). The expression level of NESTIN is also consistent with this trend (Fig. 2f, h). These results indicated that UltraGRO-medium could not only maintain but also recover the expression of NESTIN in UC-MSCs.

We then evaluated the proliferation capacity of UCMSCs under different culture conditions. The expansion ratio of the UC-MSCs cultured in three different culture conditions was calculated between the starting cell density $\left(5 \times 10^{3} / \mathrm{cm}^{2}\right.$ in a $175 \mathrm{~cm}^{2}$ flask $)$ and the cell density every 2 days. UC-MSCs cultured in UltraGRO-medium sustained their proliferation capacity with an expansion ratio above ten in each passage. However, the expansion ratio of UC-MSCs cultured in FBS-medium or TBD-FSmedium gradually decreased (Fig. 3). This result suggested that UltraGRO-medium could sustain a greater proliferation capacity of UC-MSCs than medium with the other two culture supplements.

To evaluate the immunomodulation ability of UCMSCs that highly express NESTIN, we treated the 10th passage UC-MSC in all three types of media with IFN$\gamma$, and measured the IDO and PEG2 secretion using an ELISA. IDO and PEG2 were significantly increased after IFN- $\gamma$ treatment. Furthermore, the IDO and PEG2 levels were significantly higher in the UC-MSCs cultured with UltraGRO than with the other two supplements (Fig. 4a, b). In order to analyze the immunomodulation ability in vivo, we intraperitoneally injected UC-MSCs into a trinitrobenzene sulfonic acid (TNBS)-induced colitis mouse model, and measured the proinflammatory cytokines and anti-inflammatory cytokines using an ELISA. The pro-inflammatory cytokines (IL- 6 and IL-1 $\beta$ ) were down-regulated after UC-MSC injection, and UCMSCs cultured in UltraGRO-medium showed a better inhibition effect than in the other two media (Fig. 4c, d). The anti-inflammatory cytokines (IDO and PEG2) were up-regulated simultaneously (Fig. 4e, f). This indicated that UC-MSCs may contribute to the immunomodulation against colitis and the UltraGRO group presents the best performance in immunomodulation among the three different culture conditions.

\section{Discussion}

The differentiation and proliferation capacity of UC-MSC weakens as frequent, iterated passaging increases especially after 40 - to 50 -fold population doubling (Halfon et al. 2011). Furthermore, the loss of stem cell features after the 6th passage was coincident with the decline of the mean telomere length from $9.19 \mathrm{~kb}$ to $8.7 \mathrm{~kb}$ at the 9th passage (Bonab et al. 2006). Previous studies have demonstrated that the expression of NESTIN is essential in cellular differentiation and regulation, and that it produces progenitor cells (Wiese et al. 2004; Wong et al. 2014). However, there are no studies tracking the connection between the expression of NESTIN and passaging. 

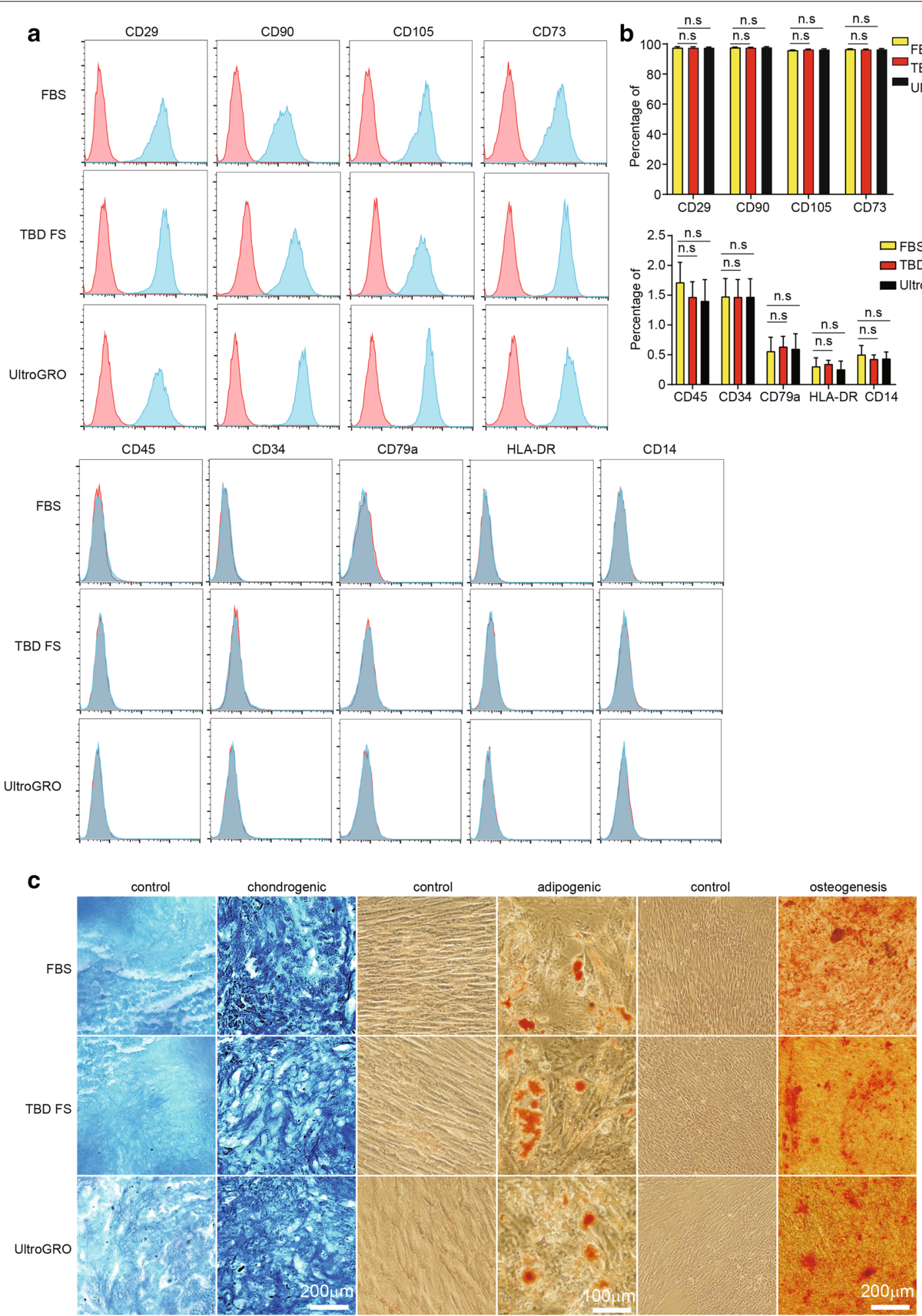

Fig. 1 The characterization of UC-MSCs in three different culture conditions. a The UC-MSCs at the 10th passage in three culture conditions were positive for CD29, CD90, CD105, and CD73, and were negative for CD45, CD34, CD79a, HLA-DR, and CD14. b The flow cytometry statistics of stem cell markers in three culture conditions. c The UC-MSCs at the 10th passage in three culture conditions were stained with Alcian blue, oil red O, and Alizarin Red $\mathrm{S}$, respectively. For all experiments, $\mathrm{n}=3$ healthy donors/group, and data shown are mean $\pm \mathrm{SD}$. 
a

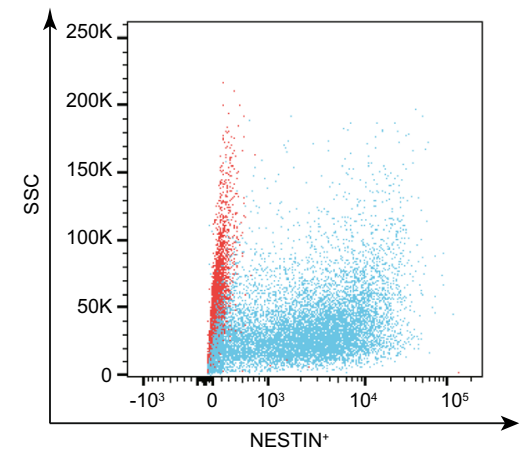

C

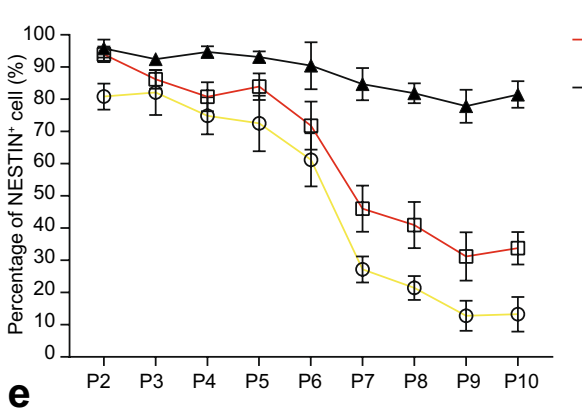

b

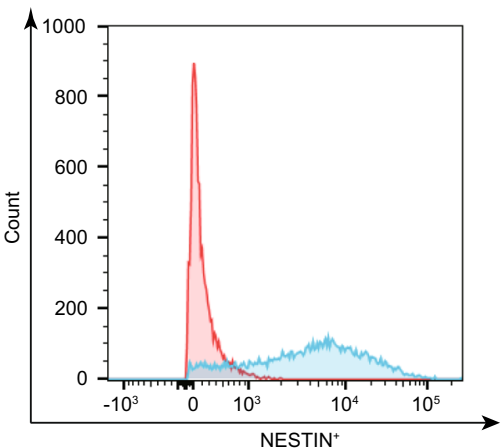

d $\square$ FBS

- FBS

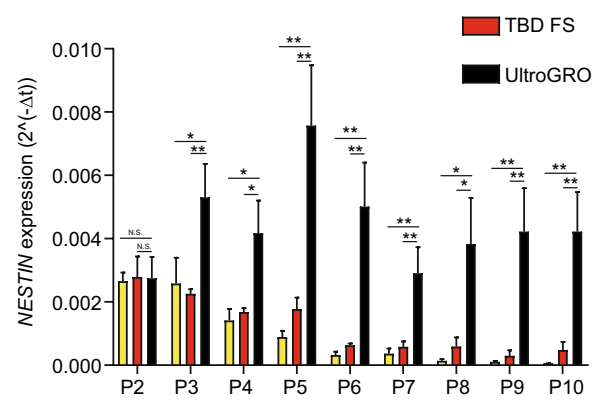

f

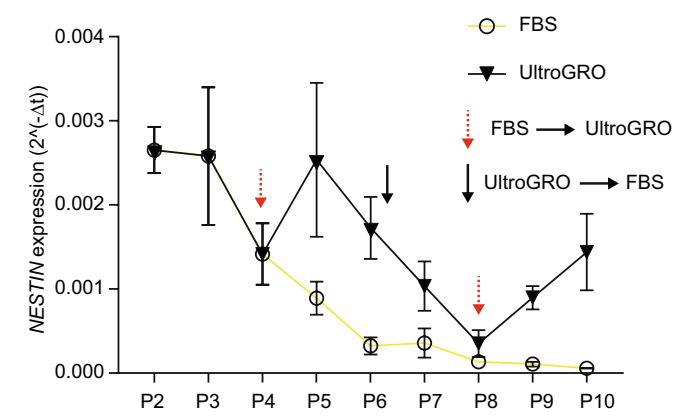

h

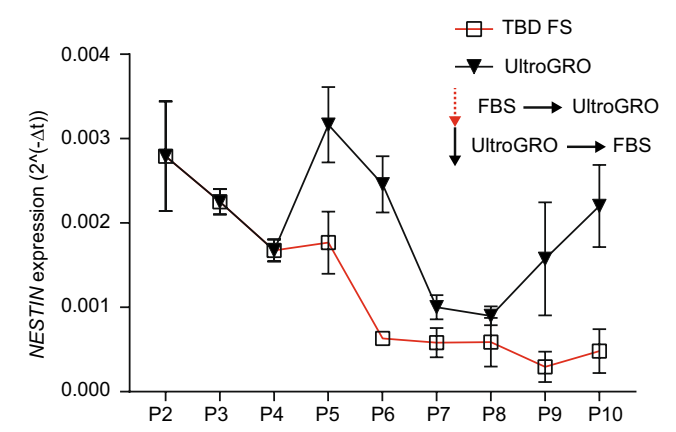

Fig. 2 The expression of NESTIN in three different culture conditions. $\mathbf{a}$, $\mathbf{b}$ The flow cytometry analysis of NESTIN in UC-MSCs. c The percentage of NESTIN ${ }^{+}$cells in three culture conditions. $\mathbf{d}$ The expression level of NESTIN in three culture conditions. $\mathbf{e}-\mathbf{h}$ The percentage of NESTIN ${ }^{+}$cells and expression level of NESTIN after changing the medium. ${ }^{* *} \mathrm{p}<0.01,{ }^{*} \mathrm{p}<0.05$, Student's $t$-test. For all experiments, $\mathrm{n}=3$ healthy donors/group, and data shown are mean \pm SD. 


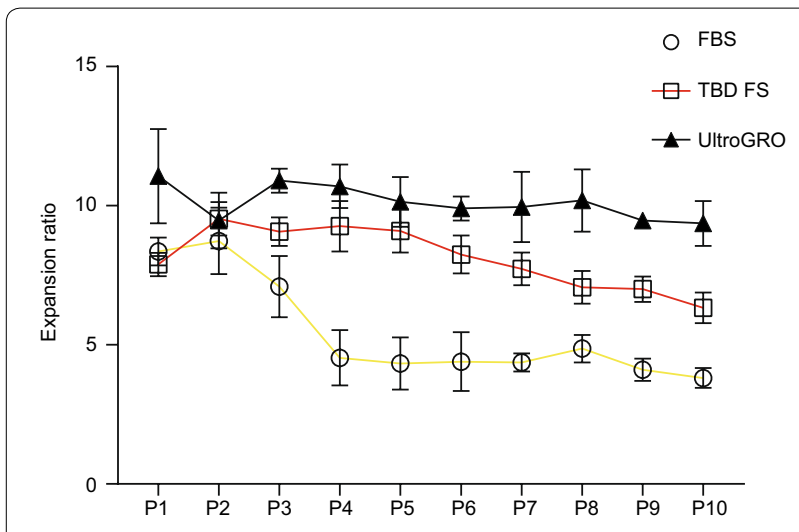

Fig. 3 The proliferation capacity of MSCs in the three groups. The proliferation ability of UC-MSCs in FBS, TBS FS, and UItraGRO group was measured by cell count. For all experiments, $n=3$ healthy donors/group, and data shown are mean $\pm S D$.

In this study, we first observed a significant change in the expression of NESTIN in UC-MSCs and the percentage of NESTIN + cells as passaging increased when cells were cultured in the conventional medium (DMEM/F12 with $10 \%$ FBS). Flow cytometry analyses for 10th passages revealed that the NESTIN ratio in either FBS or TBD FS groups experienced a significant decline after passage 6 . UltraGRO was applied in the UC-MSC culture and the NESTIN $^{+}$UC-MSCs in the UltraGRO group were maintained at a high percentage (>90\%). The NESTIN mRNA level was also in keeping with this finding. Furthermore, UltraGRO maintained the proliferation capacity of UCMSCs for up to 10 passages, likely due to certain growth factors present in the UltraGRO supplement (i.e. platelet derived growth factor, transforming growth factor, fibroblast growth factor, insulin like growth factor, etc.), cytokines, and chemokines. UltraGRO is a human single donor platelet derivative collected from healthy donors. Platelet lysate is a better than FBS and serum as a supplement for the ex vivo expansion of MSC (Bernardo et al. 2009; Bieback et al. 2009; Schallmoser et al. 2007). Stem cells proliferate via asymmetric cell division. This proliferation leads to daughter cells inheriting cellular constituents unevenly (Bernal and Arranz 2018; Gomez-Lopez et al. 2014). In addition, major morphological changes, including cytoskeletal modifications that regulate cell polarity, are required for cells to divide asymmetrically. During mitosis, the cytoskeletal protein NESTIN likely regulates the production and destruction of vimentin and other intermediate filaments (Chou et al. 2003). NESTIN may thus contribute to the abilities of self-renewal and proliferation of UC-MSCs, potentially explaining the results of the present study.

The benefit of using UC-MSCs in clinical therapy is their anti-inflammatory and immunomodulatory capabilities in a large range of diseases. UC-MSCs are a good candidate in clinical cellar therapy for treating both acute and chronic inflammatory tissue deterioration in humans and animals. UC-MSCs have immunosuppressive effects in treating Crohn's disease (CD), one of two major types of inflammatory bowel disease (Feng et al. 2018; Liao et al. 2016). Interestingly, the UC-MSC from the UltraGRO group had better anti-inflammatory performance than in other media. This suggests that the high expression of NESTIN in UC-MSC could have better immunomodulatory performance. Further investigation will be required to understand the mechanism of how NESTIN regulates the immunomodulation ability of UC-MSC.

In this study, we monitored the expression of surface markers and NESTIN for up to ten passages under three different culture conditions. Unsurprisingly, the expression of NESTIN significantly decreased in late passages using conventional medium (FBS). By comparison, the use of FBS substitute and UltraGRO can retain the expression of NESTIN, which barely decreased in the UltraGRO group after ten passages. Furthermore, UC-MSC in UltraGRO group also displays better immunomodulatory performance. However, the role of NESTIN in UC-MSC immunomodulation requires further investigation. It is believed that NESTIN could be a reporter of the status of UC-MSC during the large-scale manufacturing process, which may be used to evaluate the UC-MSC quality in stem cell therapy. 


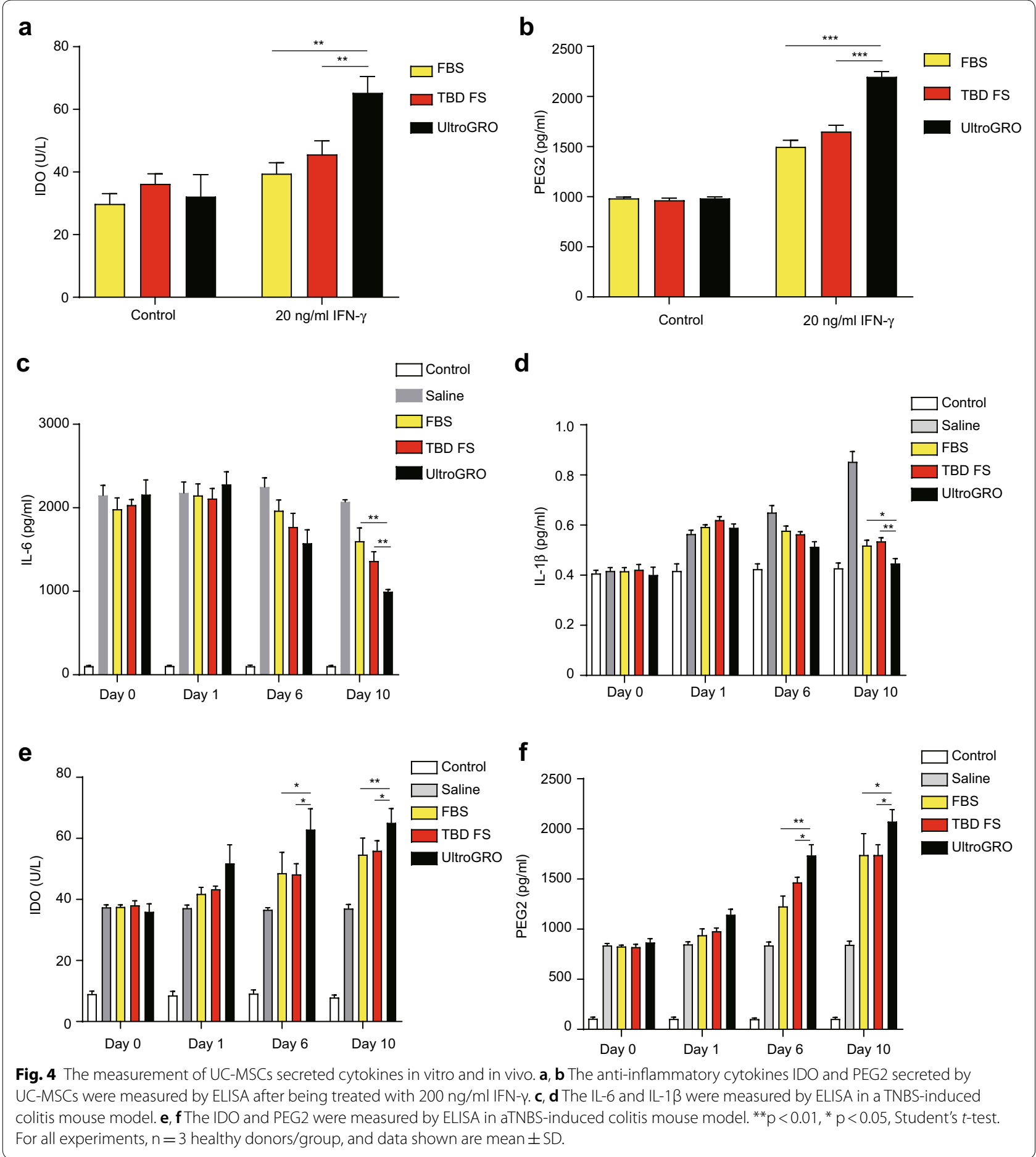

Abbreviations

MSC: Mesenchymal stem cell; ISCT: International Society of Cell Therapy; cGMP: Current good manufacture practice; UC-MSCs: Umbilical cords-mesenchymal stem cells; DMEM: Dulbecco's modified Eagle's medium; FBS: Fetal bovine serum; TNBS: Trinitrobenzene sulfonic acid; CD: Crohn's disease.

Acknowledgements

Not applicable.

\section{Authors' contributions}

$Y L$ and $F X$ conceived and designed research. $X H, M L$ and $X L$ provided research idea and sufficient resources for research. $Y L, F X$ and $Z L$ conducted experiments. FX, ZF and HP analyzed data. GZ, QM and WZ contributed new reagents or analytical tools. FX wrote the manuscript. $Z T, Y L$ and $Y R$ reviewed and revised manuscript. All authors read and approved the manuscript. 


\section{Authors' information}

$Y L$ and $F X$ are R\&D engineers from Shenzhen Beike Biotechnology Co. Ltd. Both of them have extensive experiences in the manufacture of stem cells and immune cells. Beike is a China's leading biotech company specializes in the research, clinical translation, and technological advancement of adult stem cells and their associated applications.

\section{Funding}

This work was funded by Shenzhen Beike Biotechnology Co., Ltd.

\section{Availability of data and materials}

All relevant data are within the manuscript.

\section{Ethics approval and consent to participate}

All applicable international, national, and/or institutional guidelines for the care and use of animals were followed. All of the animal procedures were reviewed and approved by the Beike Animal Care and Use Committee.

\section{Consent for publication}

Not applicable.

\section{Competing interests}

The author declares that they have no competing interests.

\section{Author details}

${ }^{1}$ National-Local Associated Engineering Laboratory for Personalized Cell Therapy, Nanshan, Shenzhen, Guangdong, People's Republic of China. ${ }^{2}$ Shenzhen Beike Biotechnology Co., Ltd., Nanshan, Shenzhen, Guangdong, People's Republic of China. ${ }^{3}$ Shenzhen Kenuo Medical Lab, Nanshan, Shenzhen, Guangdong, People's Republic of China.

Received: 26 March 2020 Accepted: 18 July 2020

Published online: 28 July 2020

\section{References}

Bernal A, Arranz L (2018) Nestin-expressing progenitor cells: function, identity and therapeutic implications. Cell Mol Life Sci 75(12):2177-2195. doi:https ://doi.org/10.1007/s00018-018-2794-z

Bernardo ME, Avanzini MA, Ciccocioppo R, Perotti C, Cometa AM, Moretta A, Marconi M, Valli M, Novara F, Bonetti F, Zuffardi O, Maccario R, Corazza GR, Locatelli F (2009) Phenotypical/functional characterization of in vitroexpanded mesenchymal stromal cells from patients with Crohn's disease. Cytotherapy 11(7):825-836. doi:https://doi.org/10.3109/1465324090 3121260

Bieback K, Hecker A, Kocaomer A, Lannert H, Schallmoser K, Strunk D, Kluter $H$ (2009) Human alternatives to fetal bovine serum for the expansion of mesenchymal stromal cells from bone marrow. Stem Cells 27(9):23312341. doi:https://doi.org/10.1002/stem.139

Bonab MM, Alimoghaddam K, Talebian F, Ghaffari SH, Ghavamzadeh A, Nikbin B (2006) Aging of mesenchymal stem cell in vitro. BMC Cell Biol 7:14. doi:https://doi.org/10.1186/1471-2121-7-14

Chinnadurai R, Copland IB, Patel SR, Galipeau J (2014) IDO-independent suppression of T cell effector function by IFN-gamma-licensed human mesenchymal stromal cells. J Immunol 192(4):1491-1501. doi:https://doi. org/10.4049/jimmunol.1301828

Chou YH, Khuon S, Herrmann H, Goldman RD (2003) Nestin promotes the phosphorylation-dependent disassembly of vimentin intermediate filaments during mitosis. Mol Biol Cell 14(4):1468-1478. doi:https://doi. org/10.1091/mbc.e02-08-0545

da Silva Meirelles L, Chagastelles PC, Nardi NB (2006) Mesenchymal stem cells reside in virtually all post-natal organs and tissues. J Cell Sci 119(Pt 11):2204-2213. doi:https://doi.org/10.1242/jcs.02932

Devito L, Klontzas ME, Cvoro A, Galleu A, Simon M, Hobbs C, Dazzi F, Mantalaris A, KhalafY, Ilic D (2019) Comparison of human isogeneic Wharton's jelly MSCs and iPSC-derived MSCs reveals differentiation-dependent metabolic responses to IFNG stimulation. Cell Death Dis 10(4):277. doi:https:// doi.org/10.1038/s41419-019-1498-0

Dominici M, Le Blanc K, Mueller I, Slaper-Cortenbach I, Marini F, Krause D, Deans R, Keating A, Prockop D, Horwitz E (2006) Minimal criteria for defining multipotent mesenchymal stromal cells. The International Society for Cellular Therapy position statement. Cytotherapy 8(4):315-317. doi:https://doi.org/10.1080/14653240600855905

Donnenberg VS, Ulrich H (2013) Mesenchymal stem cells, therapy, and cytometry. Cytometry A 83(1):8-10. doi:https://doi.org/10.1002/cyto.a.22238

Feng Y, Liao Y, Huang W, Lai X, Luo J, Du C, Lin J, Zhang Z, Qiu D, Liu Q, Shen H, Xiang AP, Zhang Q (2018) Mesenchymal stromal cells-derived matrix Gla protein contribute to the alleviation of experimental colitis. Cell Death Dis 9(6):691. doi:https://doi.org/10.1038/s41419-018-0734-3

Ferrari G, Cusella-De Angelis G, Coletta M, Paolucci E, Stornaiuolo A, Cossu G, Mavilio F (1998) Muscle regeneration by bone marrow-derived myogenic progenitors. Science 279(5356):1528-1530. doi:https://doi.org/10.1126/ science. 279.5356 .1528

Friedenstein AJ, Chailakhyan RK, Gerasimov UV (1987) Bone marrow osteogenic stem cells: in vitro cultivation and transplantation in diffusion chambers. Cell Tissue Kinet 20(3):263-272

Gomez-Lopez S, Lerner RG, Petritsch C (2014) Asymmetric cell division of stem and progenitor cells during homeostasis and cancer. Cell Mol Life Sci 71(4):575-597. doi:https://doi.org/10.1007/s00018-013-1386-1

Halfon S, Abramov N, Grinblat B, Ginis I (2011) Markers distinguishing mesenchymal stem cells from fibroblasts are downregulated with passaging. Stem Cells Dev 20(1):53-66. doi:https://doi.org/10.1089/scd.2010.0040

Kern S, Eichler H, Stoeve J, Kluter H, Bieback K (2006) Comparative analysis of mesenchymal stem cells from bone marrow, umbilical cord blood, or adipose tissue. Stem Cells 24(5):1294-1301. doi:https://doi.org/10.1634/ stemcells.2005-0342

Kulkarni S, Micci MA, Leser J, Shin C, Tang SC, Fu YY, Liu L, Li Q, Saha M, Li C, Enikolopov G, Becker L, Rakhilin N, Anderson M, Shen X, Dong X, Butte MJ, Song H, Southard-Smith EM, Kapur RP, Bogunovic M, Pasricha PJ (2017) Adult enteric nervous system in health is maintained by a dynamic balance between neuronal apoptosis and neurogenesis. Proc Natl Acad Sci USA 114(18):E3709-E3718. doi:https://doi.org/10.1073/pnas.16194 06114

Lara E, Velasquez A, Cabezas J, Rivera N, Pacha P, Rodriguez-Alvarez L, Saravia F, Castro FO (2017) Endometritis and in vitro PGE2 challenge modify properties of cattle endometrial mesenchymal stem cells and their transcriptomic profile. Stem Cells Int 2017:4297639. doi:https://doi. org/10.1155/2017/4297639

Lei J, Hui D, Huang W, Liao Y, Yang L, Liu L, Zhang Q, Qi G, Song W, Zhang Y, Xiang AP, Zhou Q (2013) Heterogeneity of the biological properties and gene expression profiles of murine bone marrow stromal cells. Int J Biochem Cell Biol 45(11):2431-2443. doi:https://doi.org/10.1016/j.bioce I.2013.07.015

Lendahl U, Zimmerman LB, McKay RD (1990) CNS stem cells express a new class of intermediate filament protein. Cell 60(4):585-595. doi:https://doi. org/10.1016/0092-8674(90)90662-X

Liao Y, Lei J, Liu M, Lin W, Hong D, Tuo Y, Jiang MH, Xia H, Wang M, Huang W, Xiang AP (2016) Mesenchymal stromal cells mitigate experimental colitis via insulin-like growth factor binding protein 7-mediated immunosuppression. Mol Ther 24(10):1860-1872. doi:https://doi.org/10.1038/ mt.2016.140

Lodie TA, Blickarz CE, Devarakonda TJ, He C, Dash AB, Clarke J, Gleneck K, Shihabuddin L, Tubo R (2002) Systematic analysis of reportedly distinct populations of multipotent bone marrow-derived stem cells reveals a lack of distinction. Tissue Eng 8(5):739-751. doi:https://doi.org/10.1089/10763 270260424105

Lu D, Liao Y, Zhu SH, Chen QC, Xie DM, Liao JJ, Feng X, Jiang MH, He W (2019) Bone-derived Nestin-positive mesenchymal stem cells improve cardiac function via recruiting cardiac endothelial cells after myocardial infarction. Stem Cell Res Ther 10(1):127. doi:https://doi.org/10.1186/s1328 7-019-1217-x

Mignone JL, Kukekov V, Chiang AS, Steindler D, Enikolopov G (2004) Neural stem and progenitor cells in nestin-GFP transgenic mice. J Comp Neurol 469(3):311-324. doi:https://doi.org/10.1002/cne.10964

Oedayrajsingh-Varma MJ, van Ham SM, Knippenberg M, Helder MN, KleinNulend J, Schouten TE, Ritt MJ, van Milligen FJ (2006) Adipose tissuederived mesenchymal stem cell yield and growth characteristics are affected by the tissue-harvesting procedure. Cytotherapy 8(2):166-177. doi:https://doi.org/10.1080/14653240600621125 
Ren G, Chen X, Dong F, Li W, Ren X, Zhang Y, Shi Y (2012) Concise review: mesenchymal stem cells and translational medicine: emerging issues. Stem Cells Transl Med 1(1):51-58. doi:https://doi.org/10.5966/sctm.2011-0019

Schallmoser K, Bartmann C, Rohde E, Reinisch A, Kashofer K, Stadelmeyer E, Drexler C, Lanzer G, Linkesch W, Strunk D (2007) Human platelet lysate can replace fetal bovine serum for clinical-scale expansion of functional mesenchymal stromal cells. Transfusion 47(8):1436-1446. doi:https://doi. org/10.1111/j.1537-2995.2007.01220.x

Seo BM, Miura M, Gronthos S, Bartold PM, Batouli S, Brahim J, Young M, Robey PG, Wang CY, Shi S (2004) Investigation of multipotent postnatal stem cells from human periodontal ligament. Lancet 364(9429):149-155. doi:https://doi.org/10.1016/S0140-6736(04)16627-0

Seo MJ, Suh SY, Bae YC, Jung JS (2005) Differentiation of human adipose stromal cells into hepatic lineage in vitro and in vivo. Biochem Biophys Res Commun 328(1):258-264. doi:https://doi.org/10.1016/j.bbrc.2004.12.158

Tondreau T, Lagneaux L, Dejeneffe M, Massy M, Mortier C, Delforge A, Bron D (2004) Bone marrow-derived mesenchymal stem cells already express specific neural proteins before any differentiation. Differentiation 72(7):319-326. doi:https://doi.org/10.1111/j.1432-0436.2004.07207003.x

Uccelli A, Moretta L, Pistoia V (2008) Mesenchymal stem cells in health and disease. Nat Rev Immunol 8(9):726-736. doi:https://doi.org/10.1038/nri2395

Uder C, Bruckner S, Winkler S, Tautenhahn HM, Christ B (2018) Mammalian MSC from selected species: features and applications. Cytometry A 93(1):32-49. doi:https://doi.org/10.1002/cyto.a.23239

Wang D, Wang S, Huang S, Zhang Z, Yuan X, Feng X, Lu L, Sun L (2017) Serum IFN-gamma predicts the therapeutic effect of mesenchymal stem cells transplantation in systemic lupus erythematosus patients. Stem Cells Transl Med 6(9):1777-1785. doi:https://doi.org/10.1002/sctm.17-0002 Wiese C, Rolletschek A, Kania G, Blyszczuk P, Tarasov KV, Tarasova Y, Wersto RP, Boheler KR, Wobus AM (2004) Nestin expression-a property of multilineage progenitor cells? Cell Mol Life Sci 61(19-20):2510-2522. doi:https ://doi.org/10.1007/s00018-004-4144-6

Wirtz S, Neufert C, Weigmann B, Neurath MF (2007) Chemically induced mouse models of intestinal inflammation. Nat Protoc 2(3):541-546. doi:https://doi.org/10.1038/nprot.2007.41

Wong A, Ghassemi E, Yellowley CE (2014) Nestin expression in mesenchymal stromal cells: regulation by hypoxia and osteogenesis. BMC Vet Res 10:173. doi:https://doi.org/10.1186/s12917-014-0173-z

Zimmerman L, Parr B, Lendahl U, Cunningham M, McKay R, Gavin B, Mann J, Vassileva G, McMahon A (1994) Independent regulatory elements in the nestin gene direct transgene expression to neural stem cells or muscle precursors. Neuron 12(1):11-24. doi:https://doi.org/10.1016/08966273(94)90148-1

Zuk PA, Zhu M, Mizuno H, Huang J, Futrell JW, Katz AJ, Benhaim P, Lorenz HP, Hedrick MH (2001) Multilineage cells from human adipose tissue: implications for cell-based therapies. Tissue Eng 7(2):211-228. doi:https://doi. org/10.1089/107632701300062859

\section{Publisher's Note}

Springer Nature remains neutral with regard to jurisdictional claims in published maps and institutional affiliations.

\section{Submit your manuscript to a SpringerOpen ${ }^{\odot}$ journal and benefit from:}

- Convenient online submission

- Rigorous peer review

- Open access: articles freely available online

- High visibility within the field

- Retaining the copyright to your article

Submit your next manuscript at $\boldsymbol{\Delta}$ springeropen.com 